\title{
Simulation of optical properties of Si photonic crystals
}

\author{
K. A. I. L. Wijewardena Gamalath*, W. A. S. C. Settinayake \\ Department of Physics, University of Colombo, Colombo 03, Sri Lanka \\ *E-mail address: Imalie@phys.cmb.ac.Ik
}

\begin{abstract}
To investigate optical properties of Si photonic crystal waveguides, a mathematical model was set up. Finite difference time domain method was used to calculate the Maxwell's equations numerically. For the evolution of the electromagnetic fields in the photonic crystals, simulations were done for a small lattices using Yee lattice approach. The properties of a waveguide and a power divider were investigated for $3 \lambda \times 3 \lambda$ photonic crystal formed from $\mathrm{Si}$ circular rods in air for telecommunication wavelength $1.55 \mu \mathrm{m}$. The model developed was satisfactory in predicting the behaviour of light in linear photonic crystals.
\end{abstract}

Keywords: Photonic crystal; waveguides; power divider; Finite difference time domain method; Yee lattice approach

\section{INTRODUCTION}

In the last two decades, a new frontier to engineer materials that are capable of controlling light known as photonic crystals have emerged. These crystals which are periodic structures with periodicity in micrometer to millimetre scale are artificially arranged so as to tailor light and propagate electromagnetic waves in a unique way. In analogy to electrons in a semiconductor crystal, the electromagnetic waves propagating in a structure with a periodically modulated dielectric constant are organized into photonic bands which are separated by gaps where propagating states are forbidden. These artificial periodic dielectric structures are employed in a systematic way to achieve a band gap in a desired wavelength [1]. Since there is no quantum mechanical state for a photon, the light is not emitted from the source, reflected back by the crystal and eventually re-absorbed or re-emitted. Thus photonic crystals can be used as perfect reflectors. The photonic band gap materials have unusual dispersion properties that strongly influence the propagation characteristics of light beams. Photonic crystals, which prohibit the propagation of light for frequencies within a band gap, have enabled exciting new ways to control light and construct integrated optical devices. Among the most basic optical components for integrated optics applications are linear waveguides and Y splitters.

There are many methods available for the study of the behavior of light inside a photonic crystal, finite difference time domain method (FDTD) been one. The basic algorithm of this method was found in 1966 by Kane Yee [2] and has been extensively used in the last few years due to the introduction of fast computers such as in modeling the electric 
fields inside antennas and radar systems. The high refractive index of silicon makes it an ideal component for the creation of photonic structures with strong light confinement. Si photonic wire waveguides are attractive for constructing various optical devices that are extremely small because the waveguides can be bent with extremely small curvatures of less than a few micrometers of bending radius.

In the present study, the optical properties of silicon photonic crystal waveguides and power divider were investigated by finite difference time domain method. Simulations were done for a small lattice by calculating the Maxwell's equations numerically for a predetermined grid points. Using this method, the grid selected need not be restricted to Cartesian rectangular space nor is limited to modeling light. Sound can also be modeled by using this method with the replacement of relevant parameters. The main advantage is that there is no need for symmetry but it is practical only for small grids, otherwise the computational domain increases and much insight cannot be obtained at far regions from the source. A pulsed optical source is assumed to be located at one end of photonic crystal. Using Yee lattice approach the transmission power and the transmission coefficients were calculated by numerically integrating the power output of all the points in the detectors for these photonic crystals structures.

\section{ELECTRIC AND MAGNETIC FIELDS INSIDE PHOTONIC CRYSTALS}

The propagation of light inside a photonic crystal is governed by four macroscopic Maxwell equations. For electromagnetic waves in a macroscopic, homogeneous, isotropic dielectric material with no charges and sources $(\rho=0, J=0)$ Maxwell's equations of Faraday's law of induction and Ampere's law for electric field $\boldsymbol{E}$ and magnetic field strength $\boldsymbol{H}$ in SI units are

$$
\nabla \wedge \boldsymbol{E}=-\mu(r) \frac{\partial \boldsymbol{H}}{\partial t}, \quad \nabla \wedge \boldsymbol{H}=\varepsilon(r) \frac{\partial \boldsymbol{E}}{\partial t}+\boldsymbol{J}
$$

respectively. Where $\boldsymbol{J}$ is the current density, $\varepsilon(r)$ and $\mu(r)$ are position dependent electric permeability and magnetic permittivity respectively.

If the material considered has no dispersion, the dielectric constant $\varepsilon(r)$ is purely real and for most dielectric materials $\mu(r) \approx 1$. Therefore $\mu(r)$ is assumed to be a constant. Let

$$
\tilde{E}(\boldsymbol{r}, t)=\sqrt{\frac{\varepsilon_{0}}{\mu_{0}}} E(\boldsymbol{r}, t), \quad \tilde{D}(\boldsymbol{r}, t)=\frac{1}{\sqrt{\mu_{0} \varepsilon_{0}}} D(\boldsymbol{r}, t)
$$

where $\varepsilon_{0}$ is the permittivity and $\mu_{0}$ the permeability in free space. Equations in 1 now read:

$$
\frac{1}{\sqrt{\mu_{0} \varepsilon_{0}}} \nabla \wedge H(\boldsymbol{r}, t)=\frac{\partial}{\partial t} \tilde{D}(\boldsymbol{r}, t), \quad \frac{1}{\sqrt{\mu_{0} \varepsilon_{0}}} \nabla \wedge \tilde{E}(\boldsymbol{r}, t)=-H(\boldsymbol{r}, t)
$$


In two dimensions, these equations can be broken up into transverse electric (TE) and transverse magnetic (TM) modes. If the simulation plane is parallel to the $x y$ plane, then the field equations governing the TM mode with $H_{z}=0, E_{z} \neq 0$ can be written as

$$
\frac{\partial D_{z}}{\partial t}=\frac{1}{\sqrt{\mu_{0} \varepsilon_{0}}}\left(\frac{\partial H_{y}}{\partial x}-\frac{\partial H_{x}}{\partial y}\right), \quad \frac{\partial H_{x}}{\partial t}=-\frac{1}{\sqrt{\mu_{0} \varepsilon_{0}}} \frac{\partial E_{z}}{\partial y}, \quad \frac{\partial H_{y}}{\partial t}=-\frac{1}{\sqrt{\mu_{0} \varepsilon_{0}}} \frac{\partial E_{z}}{\partial x}
$$

In order to obtain $E_{z}$ values, simultaneous equations have to be solved. To avoid this problem a Yee cell shown in Figure 1 is used. Here $\boldsymbol{E}$ fields are calculated in one time step and the $\boldsymbol{H}$ field values in the other. This method is continued with small time steps. By denoting the time step as superscript and the spatial grid position of the field as $i$ and $j$ arguments in parentheses, the set of equations now reduces to

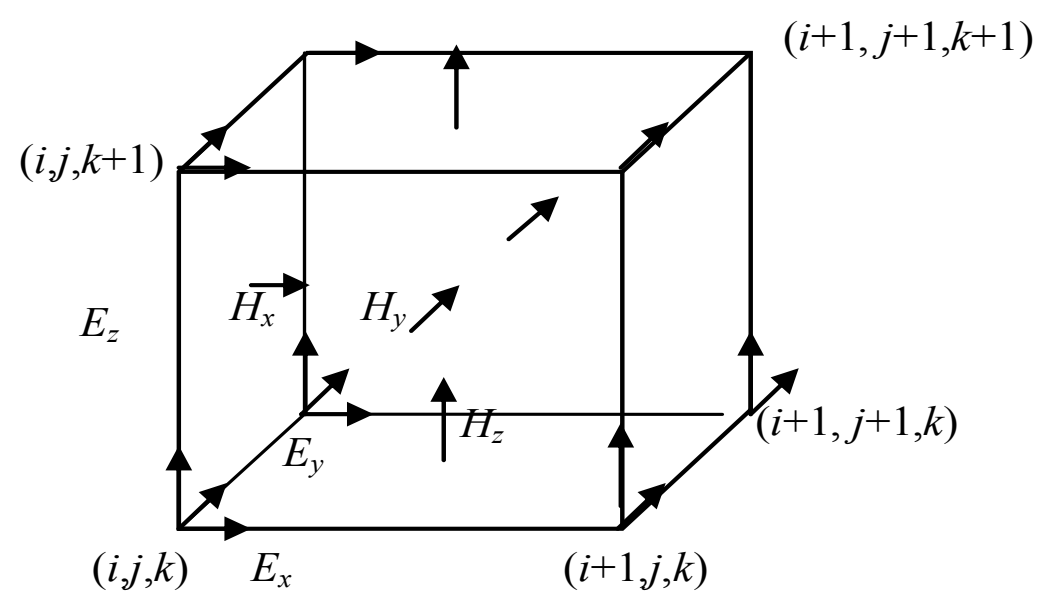

Figure 1. The Yee lattice.

$$
\begin{gathered}
\frac{D_{z}^{\left(n+\frac{1}{2}\right)}(i, j)-D_{z}^{\left(n-\frac{1}{2}\right)}(i, j)}{\Delta t}=\frac{1}{\sqrt{\mu_{0} \varepsilon_{0}}} \frac{H_{y}^{n}\left(i+\frac{1}{2}, j\right)-H_{y}^{n}\left(i-\frac{1}{2}, j\right)}{\Delta x}-\frac{1}{\sqrt{\mu_{0} \varepsilon_{0}}} \frac{\left(H_{x}^{n}\left(i, j+\frac{1}{2}\right)-H_{x}^{n}\left(i, j-\frac{1}{2}\right)\right.}{\Delta y} \\
\frac{H_{x}^{n+1}\left(i, j+\frac{1}{2}\right)-H_{x}^{n}\left(i, j+\frac{1}{2}\right)}{\Delta t}=-\frac{1}{\sqrt{\mu_{0} \epsilon_{0}}} \frac{E_{z}^{n+\frac{1}{2}}(i, j+1)-E_{z}^{n+\frac{1}{2}}(i, j)}{\Delta \mathrm{y}} \\
\frac{H_{y}^{n+1}\left(i+\frac{1}{2}, j\right)-H_{y}^{n}\left(i+\frac{1}{2}, j\right)}{\Delta t}=-\frac{1}{\sqrt{\mu_{0} \epsilon_{0}}} \frac{E_{z}^{n+\frac{1}{2}}(i+1, j)-E_{z}^{n+\frac{1}{2}}(i, j)}{\Delta \mathrm{x}}
\end{gathered}
$$


The magnetic field is calculated at half intervals from the electric field in both space and time. From this, the final equations for the calculation of $E$ field values and $H$ field values are:

$$
\begin{aligned}
& D_{z}^{n+\frac{1}{2}}(i, j)=D_{z}^{n-\frac{1}{2}}(i, j)+\frac{\Delta t}{\sqrt{\mu_{0} \epsilon_{0}}}\left(\frac{H_{y}^{n}\left(i+\frac{1}{2}, j\right)-H_{y}^{n}\left(i-\frac{1}{2}, j\right)}{\Delta \mathrm{x}}\right)-\frac{\Delta t}{\sqrt{\mu_{0} \epsilon_{0}}}\left(\frac{H_{x}^{n}\left(i, j+\frac{1}{2}\right)-H_{x}^{n}\left(i, j-\frac{1}{2}\right)}{\Delta \mathrm{y}}\right) \\
& H_{x}^{n+1}\left(i, j+\frac{1}{2}\right)=H_{x}^{n}\left(i, j+\frac{1}{2}\right)-\frac{\Delta t}{\sqrt{\mu_{0} \epsilon_{0}}}\left(\frac{E_{z}^{n+\frac{1}{2}}(i, j+1)-E_{z}^{n+\frac{1}{2}}(i, j)}{\Delta \mathrm{y}}\right) \\
& H_{y}^{n+1}\left(i+\frac{1}{2}, j\right)=H_{y}^{n}\left(i+\frac{1}{2}, j\right)-\frac{\Delta t}{\sqrt{\mu_{0} \epsilon_{0}}}\left(\frac{E_{z}^{n+\frac{1}{2}}(i+1, j)-E_{z}^{n+\frac{1}{2}}(i, j)}{\Delta \mathrm{x}}\right)
\end{aligned}
$$

where $\Delta x$ and $\Delta y$ are lattice increments in $x$ and $y$ directions. As the changes in the electromagnetic field must not propagate faster than the speed of light, for a $2 \mathrm{D}$ crystal, the time step must satisfy the condition,

$$
\Delta t \geq \frac{1}{c}\left(\frac{1}{d x^{2}}+\frac{1}{d y^{2}}\right)
$$

This is called the Courant condition [3]. Furthermore cell size should avoid Nyquist limit by a comfortable margin. That is the wavelength of electromagnetic radiation $\lambda=2 \times d x$ . Similar equations can be derived for TE polarization.

\section{TRANSMISSION COEFFICIENT}

A three dimensional crystal with a varying refractive index can be approximated to a three dimensional space with different values of electric permittivity and magnetic permeability at different coordinates. Inside a dielectric, the induced polarization $P$ of the medium is given by the optical Kerr effect

$$
\begin{aligned}
& D=\varepsilon_{0} E+P \\
& \mathrm{P}=\varepsilon_{o} \chi^{(1)} E+\varepsilon_{o} \chi^{(2)} E^{2}+\varepsilon_{o} \chi^{(3)} E^{3}+\cdots
\end{aligned}
$$

For linear optics, the second and third order electric susceptibilities of the medium $\chi^{(2)}, \chi^{(3)}$ are considered to be negligible whereas for non linear optics, second order electric susceptibility $\chi^{(2)}=0$ due to symmetry. The index of refraction $n$ is given by

$$
n^{2}=1+\chi^{(1)}
$$


Because of their symmetries, the isotropic materials have a single refractive index. For an electric field $E=E_{\omega} \cos \omega t$, the induced polarization of the medium now read

$$
P \approx \varepsilon_{0}\left(\chi^{(1)}+\frac{3}{4} \chi^{(3)}\left|E_{\omega}\right|^{2}\right) E_{\omega} \cos \omega t
$$

and the index of refraction

$$
\begin{aligned}
& n=(1+\chi)^{\frac{1}{2}}=\left(1+\chi_{\text {Linear }}+\chi_{\text {NonLinear }}\right)^{\frac{1}{2}} \\
& n \approx\left(1+\frac{1 \times \chi_{\text {Linear }}}{2 n_{0}{ }^{2}}\right)=n_{0}+\left(\frac{3 \chi^{(3)}}{8 n_{0}}\right) \times\left|E_{\omega}\right|^{2}=n_{0}+n_{2} I
\end{aligned}
$$

where $I$ is the intensity of light. The value of $n_{2}$ is usually small and a significant change in $n$ can be obtained by high powered laser beams in the order of $10^{-10}$. The electric field and displacement fields are now related by,

$$
E(t)=\frac{D(t)}{\varepsilon_{0}\left(1+\chi^{(1)}+\frac{3}{4} \chi^{(3)}|E(t)|^{2}\right)}
$$

However it should be noted that this approach can only be carried out for TE waves where all the electric field values are in one direction. Otherwise the crystal polarizes differently in different directions and $\chi$ becomes a tensor. The equation for the energy intensity of light inside a dielectric material is

$$
I=\frac{1}{2} \varepsilon v E_{0}^{2}
$$

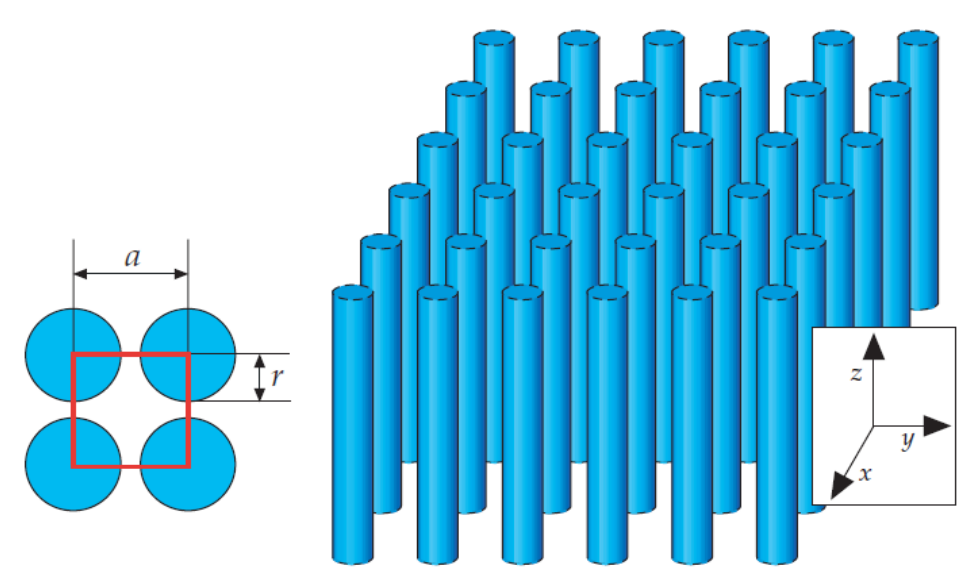

Figure 2(a). two dimensional photonic crystal of square lattice of dielectric circular rods. The unit cell at the left is framed red. 


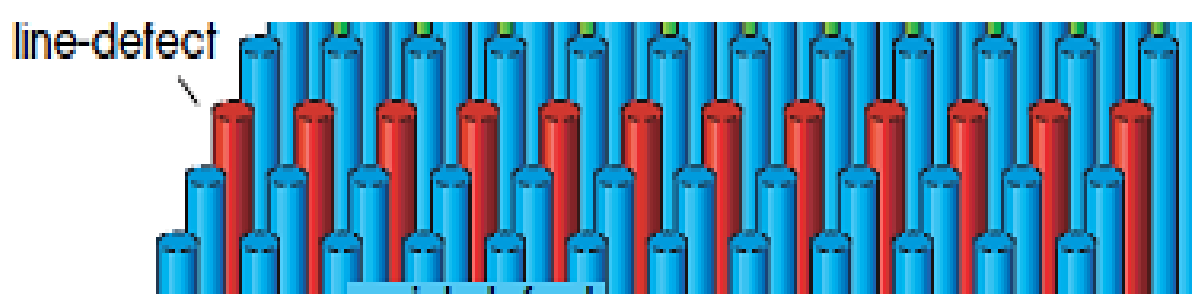

Figure 2(b). Perturbing one row in the bulk crystal shown in red [1].

By numerically integrating the power output of all the points in a detector it is possible to find the total power output. Once we find the point with maximum power we can compare that with that of the input to get the transmission coefficient.

Due to spatial periodicity in dielectric constant of photonic crystal materials, the interaction between electron and the periodic potential result in the formation of energy bandgaps. In order for a particle to interact with its periodic environment, its wavelength must be comparable to the periodicity of the lattice.

Therefore, for photonic crystals, the lattice constant must be in the range of $100 \mathrm{~nm}-1$ $\mu \mathrm{m}$. Although it is not possible for the electron to pass through the crystal if its energy falls in the range of the bandgap, defects or a break in the periodicity of the lattice can locally destroy the bandgap giving rise to interesting electronic properties. While photons with energies within the photonic bandgap cannot propagate through the crystal, they can be confined to defect regions. Light with a frequency within the bandgap can only propagate along the channel of defects since it is repelled from the bulk crystal.

\section{PHOTONIC CRYSTAL WAVEGUIDE}

An important element of optical circuits is a linear waveguide which carry light to and from components. The photonic crystals provide unique advantages for waveguides. In an optical waveguide, the light localized by introducing linear defects can be guided from one location to another. A waveguide can be carved out of a perfect photonic crystal by removing a linear sequence of unit cells as shown in Figure 2. Light that propagates in the waveguide with a frequency within the band gap is confined to the defect based on total reflection phenomena at the interfaces and can be directed along the defect.

Since silicon photonics is experiencing a dramatic increase in interest due to emerging application areas and several high profile successes in device and technology development, a square lattice of size $3 \lambda \times 3 \lambda$ ( $\lambda$ wavelength) formed from silicon circular rods of $1 \mu \mathrm{m}$ radius in air was used for all the simulations. A line defect in y direction was created by removing a layer of rods to form a straight waveguide. The crystal structure and the placement of the laser source and the detector for a straight waveguide are shown in Figure 3. A perfectly matching layer was implemented at the boundaries to avoid reflections at the boundaries. The simulated grid length was taken as one eightieth of the wavelength $(\lambda / 80)$.

The index of refraction of Si rods was taken as 12 and air as 1 and the refractive index of the each point was changed according to equation 18 in every time step of the finite difference time domain simulation $[4,5]$. 


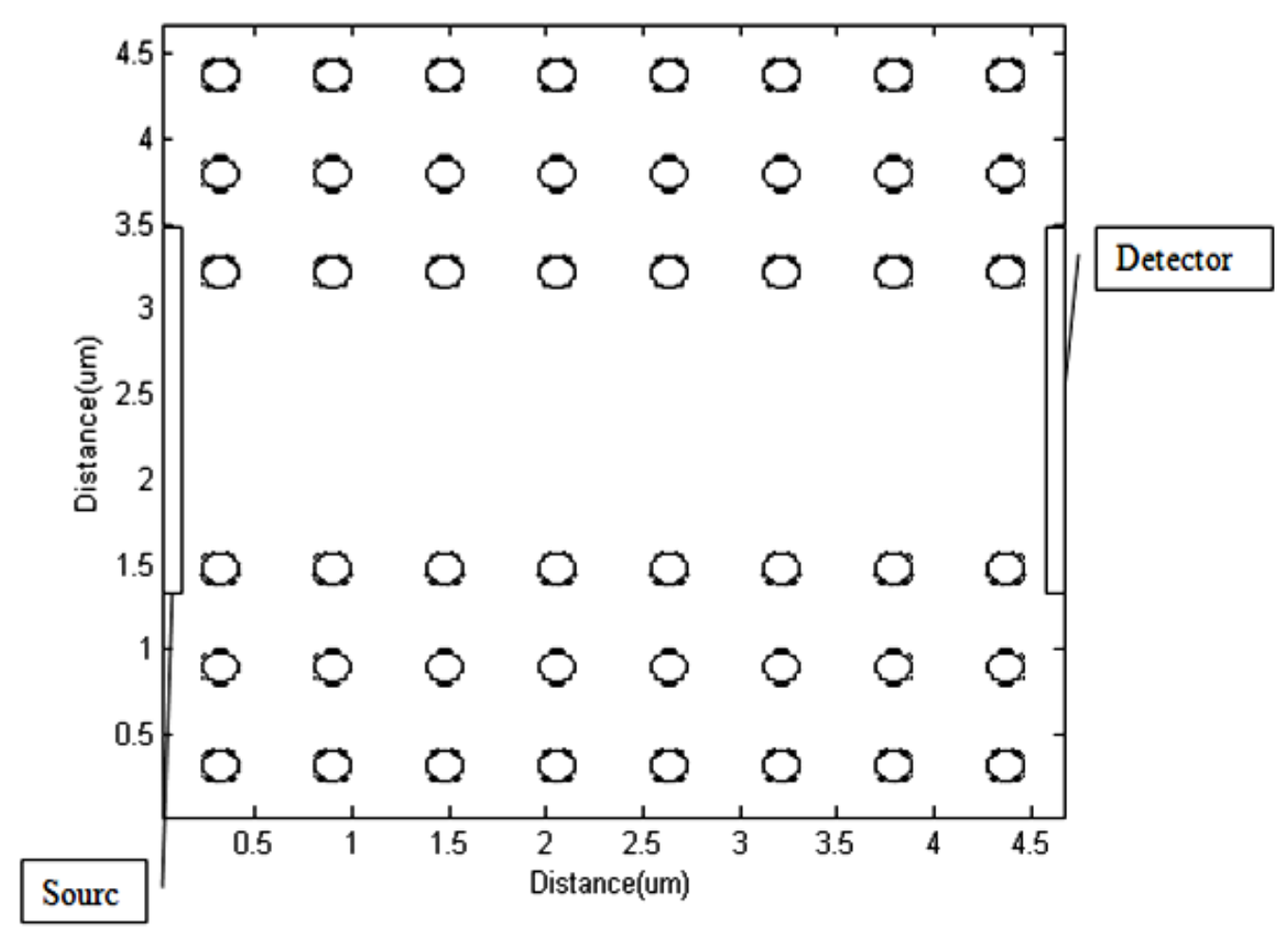

Figure 3. The rod arrangement in the crystal for the straight waveguide.

We have assumed that the structure is infinite in y and z-directions. Since for computational purposes this had to be made finite, the reflections at the boundaries were avoided by setting the field values artificially to zero by using perfectly matching layers for the crystal boundaries, thereby the crystal appeared unbounded for the simulation.

The polarization of the incident wave to the structure, can be divided into two parts of TE ( $H$ polarization), where the $E$-field is in a plane normal to the infinite axis of the dielectric rods and TM ( $E$ polarization), which $E$-field is parallel to the axis of rods. A laser pulse of wavelength $1.55 \mu \mathrm{m}$ utilized in telecommunications was sent along the defect of the crystal and the resultant intensity was measured at the other end. The source used is a hard source describing a simple sinusoidal wave so that the incident electric field from the source does not add on to the value but replaces it completely. Finite difference time domain FDTD method was used for the analysis of electromagnetic field inside the photonic crystal. All the electric field values and the magnetic field values for all the points in the crystal at different times were numerically calculated. Any initial field distribution, which may be generated at the beginning of the waveguide, can be decomposed into a linear combination of the field distributions of the guided waveguide modes.

The electric field recorded for fifty iterations inside the crystal for different time steps are shown in Figure 4. For this system of linear defect, the discrete translation symmetry is preserved in y-direction and the wave vector in y-direction $\left(k_{y}\right)$ is still conserved and we maintain translation symmetry in z-direction and restrict to the propagation in xy-plane $\left(k_{z}=\right.$ 0 ) and consider only TM polarization ( $E_{z}$ only). Wherein the introduction of the defect, result in a discrete guided band lying inside the TM band gap. By virtue of the band gap, the mode represented by this band is evanescent within the crystal and is localized within the defect. 

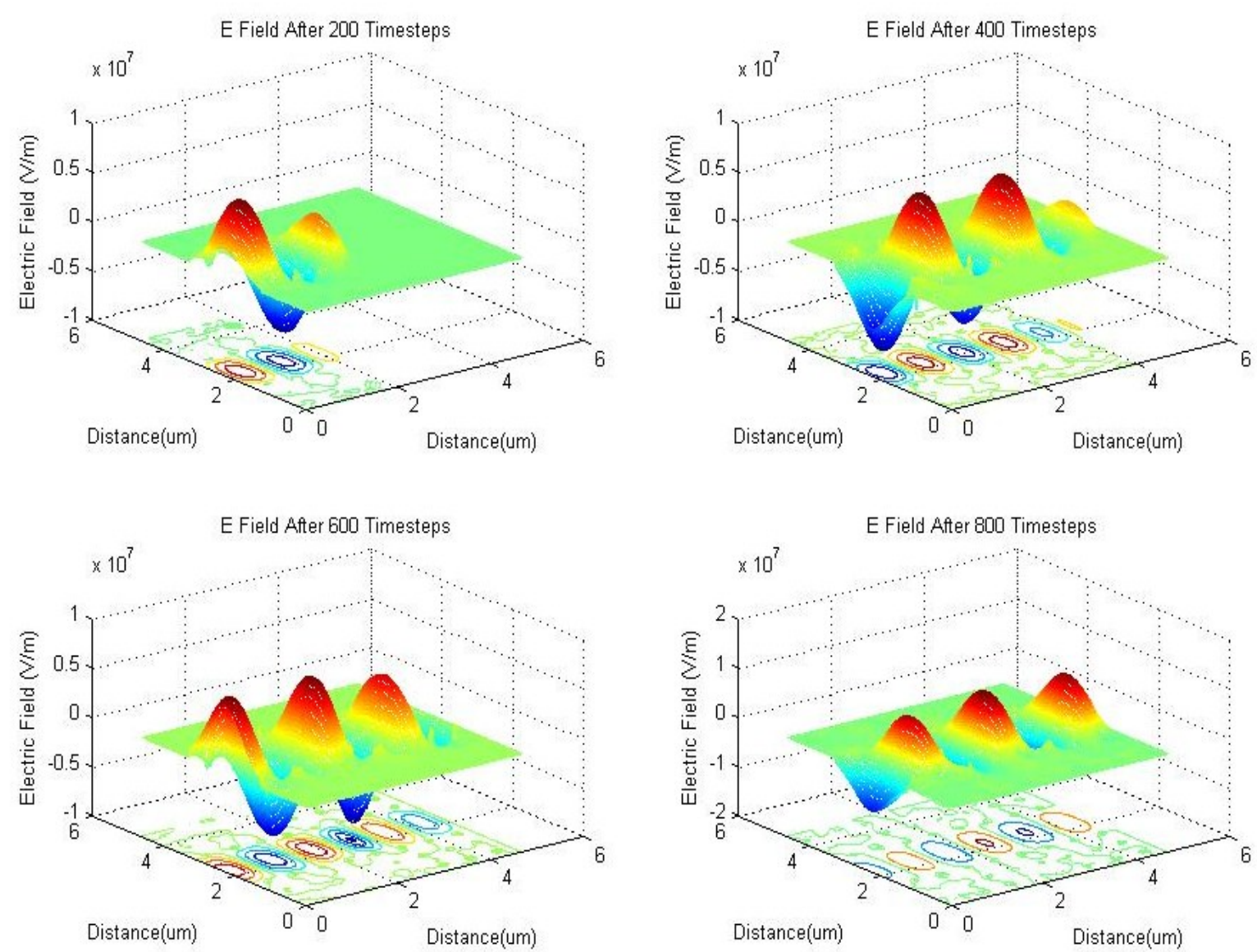

Figure 4. The electric field inside the crystal at different times for straight wave guide.
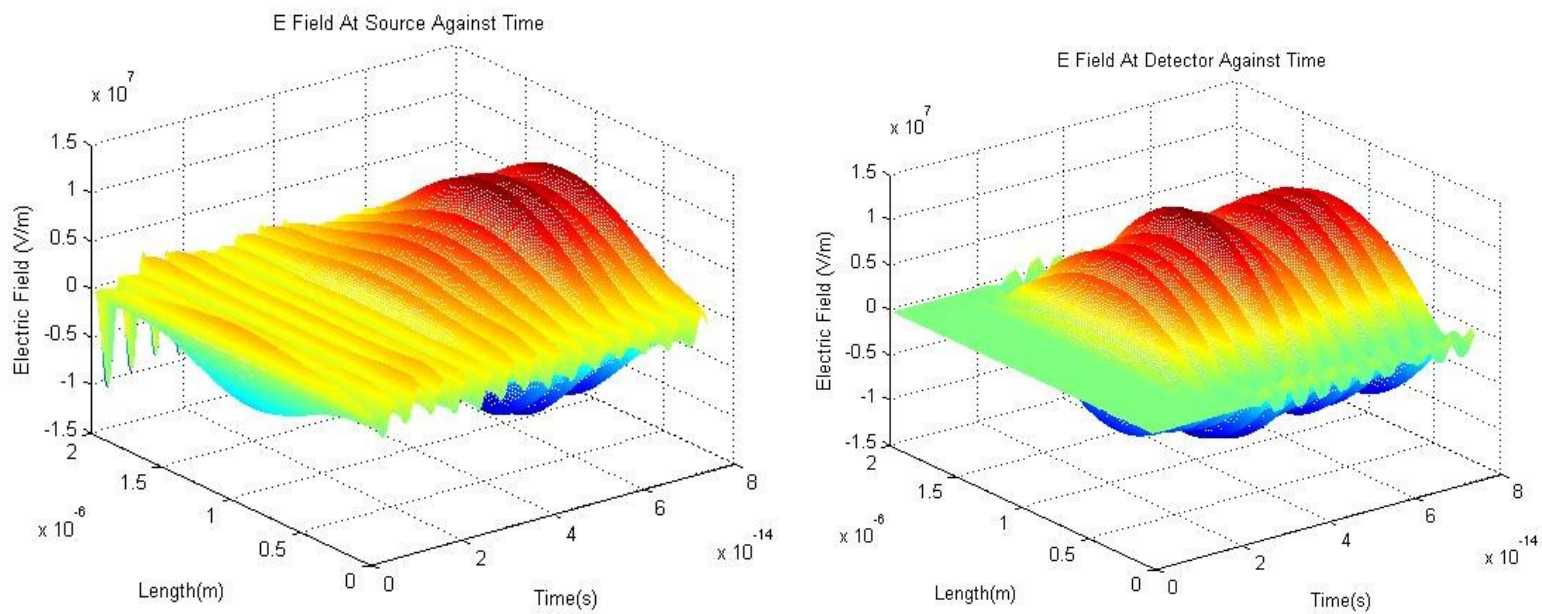

Figure 5. The electric field at the source (left) and at the detector (right) for the straight waveguide.

There is only one guided band and the waveguide is single mode. The wave motion inside the waveguide is clearly visible in the Figure 4. A closer look shows that the electric field intensity is increased in the middle of the defect or light and focused at the source 
(Figure 4). The electric field against time for the source and the detector are shown in Figure 5 . The total input power and the output power were calculated by numerically integrating the power at all point of the source and detector. The transmission coefficient calculated was 0 . 905. Because of the artificially introduced source, the reflected rays come back, get amplified and hence caused an exponential increase in electric field near the source.

\section{POWER DIVIDER}

A power divider is ideally a lossless reciprocal device which can perform vector summation of two or more signals and thus is sometimes called a power combiner. In typical power-splitting applications, the input power is divided into a number of smaller amounts for exciting the radiating elements in an array antenna. For the same photonic crystal made of $\mathrm{Si}$ circular rods of radius of $1 \mu \mathrm{m}$ in air, a power divider was formed by creating two line defects in the shape of a letter Y inside the crystal as shown in Figure 6.

Two detectors were placed at two ends and the crystal structure and the placements of the laser source and the detector are shown in this figure. A laser pulse of wavelength 1.55 $\mu \mathrm{m}$ was sent through the other end. The electric field inside the crystal for different time steps of are shown in Figure 7 and at the source and two detectors in Figure 8 respectively. Both output powers were identical and only $43 \%$ of the input power reached to any of the two output ports. The number of time steps was increased to 1600 .

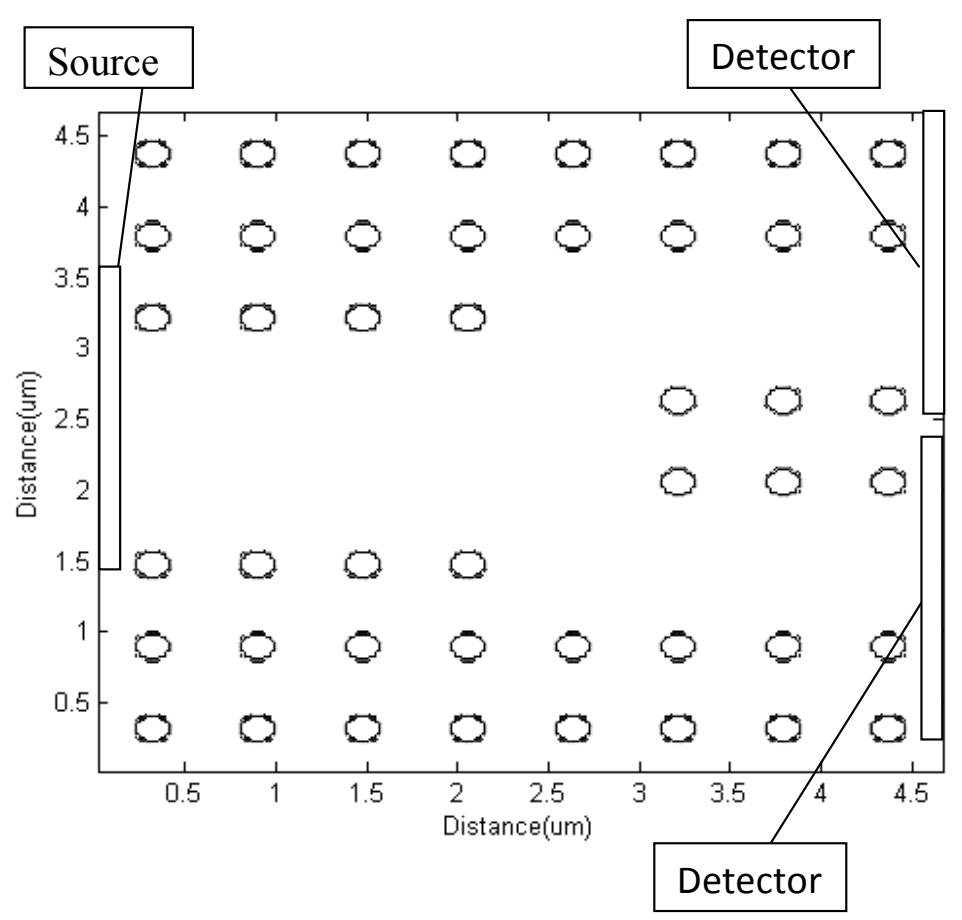

Figure 6. The rod arrangement in the crystal for splitting the laser beam. 

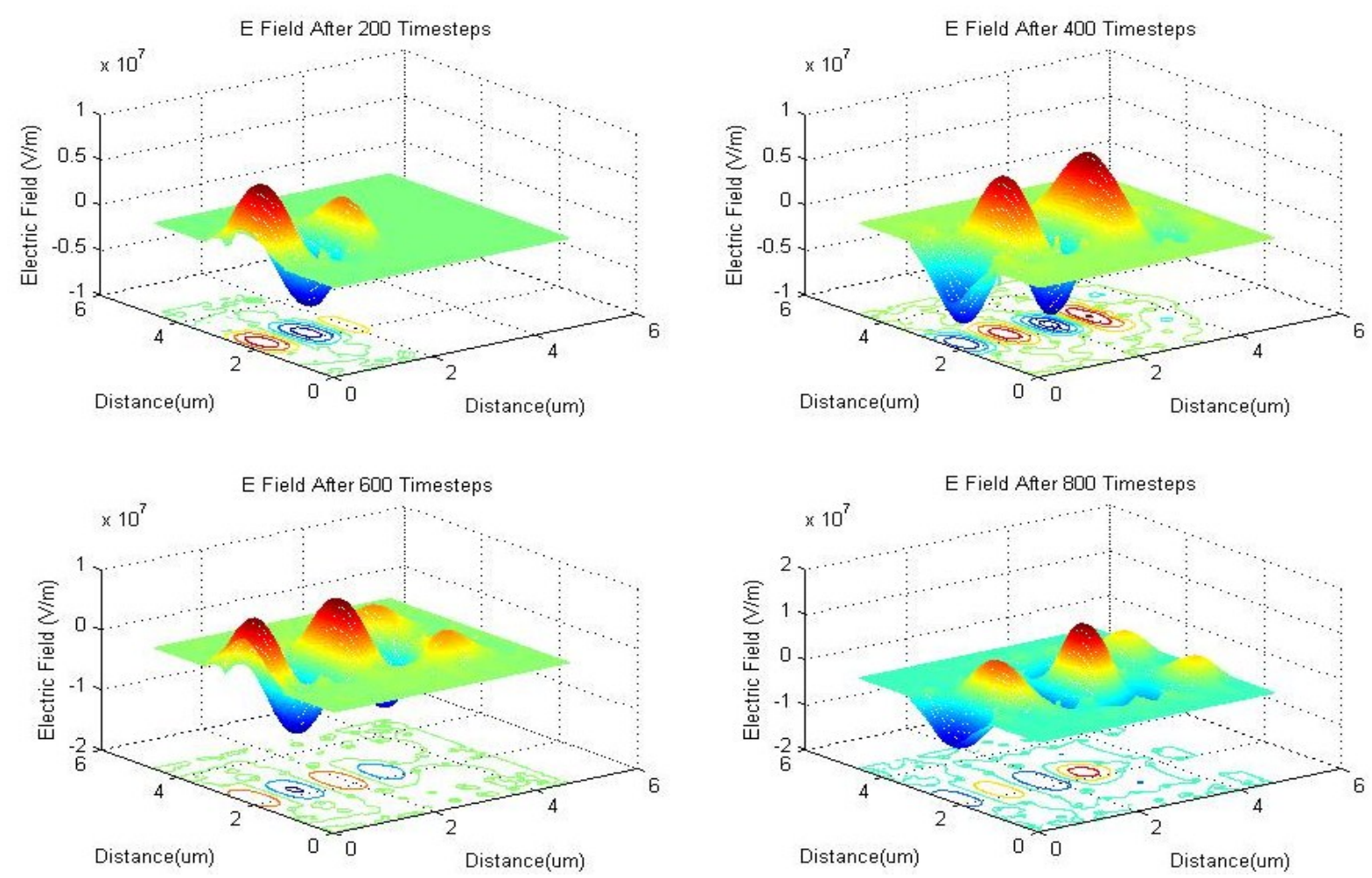

Figure 7(a). Electric field values in the crystal at different times in the wave splitter.
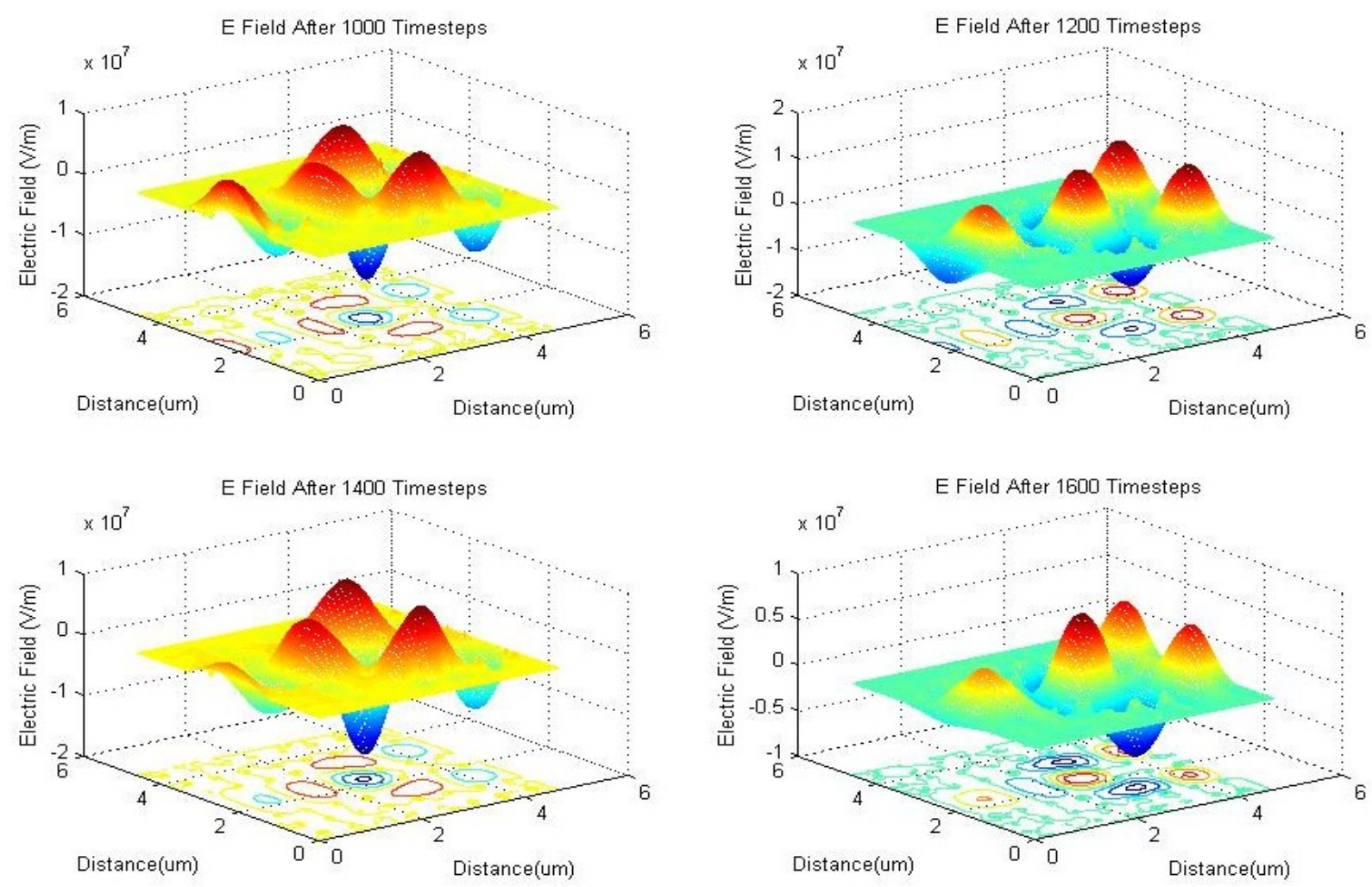

Figure 7(b). Electric field values in the crystal at different times in the wave splitter 

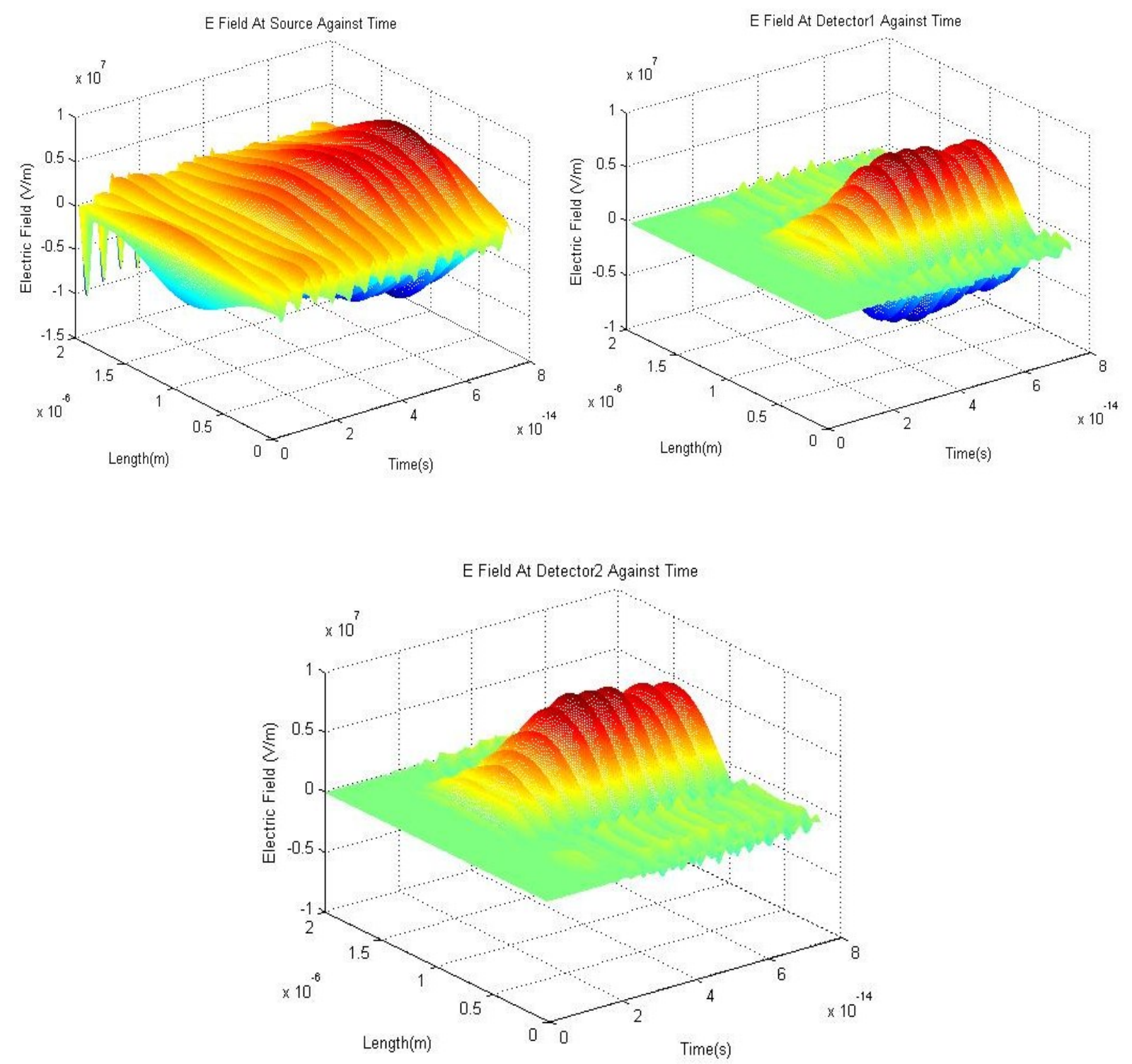

Figure 8. The electric field at the source and two detectors for the wave splitter.

\section{CONCLUSION}

The waveguide offers an efficient media for nonlinear optical functions. For the simulation of the photonic crystal formed from Si circular rods of radius of $1 \mu \mathrm{m}$ in air with line defects created by removing rods, gave a transmission of $90.5 \%$ for a straight waveguide while the power divider split the power of the input signal into two equal powers with a total of $86 \%$ transmission for the telecommunication wave length $1.55 \mu \mathrm{m}$. The wave motion inside the waveguide and power splitter is clearly visible indicating single mode transmissions. We have demonstrated the effectiveness of the model by simulating common designs of the waveguide and power divider. 


\section{References}

[1] J. D. Joannopoulos, Photonic Crystals Moulding The Flow Of Light. Princeton University Press (2008).

[2] Kane Yee, IEEE Transactions on 14 (1966) 302-307.

[3] N. Nozhat, N. Granpayeh, J. App. Sci. 7(22) (2007) 3576-3579.

[4] Hyun-Ho Lee, Kyu-Min Chae, Sang-Youp Yim, Seung-Han Park, Optics Express 12 (2004) 2603-2609.

[5] S. E. Dissanayake, K. A. I. L. Wijewardena Gamalath, International Letters of Chemistry, Physics and Astronomy 5 (2014) 58-88. 